\title{
APPLICATIONS OF CLUSTER SETS IN MINIMAL TOPOLOGICAL SPACES
}

\author{
T. R. HAMLETT
}

ABSTRACT. Given a function $f$ from a topological space $X$ into a topological space $Y$ and a point $x \in X$, the cluster set of $f$ at $x$ is $\mathcal{C}(f ; x)=\bigcap\{\mathrm{Cl}(f(U)): U$ is a neighborhood of $x\}$, where $\mathrm{Cl}(U)$ denotes the closure of $U$. In this paper, $Y$ is taken to be a minimal topological space and $C(f ; x)$ is used as a tool to obtain information about the continuity of $f$.

1. Introduction. If $X$ is a topological space and $x \in X$, let $\Re(x)$ denote the nbd (neighborhood) system at $x$. Given a function $f$ from a topological space $X$ into a topological space $Y$, J. D. Weston [6] defined the cluster set of $f$ at $x \in X$ to be

$$
\mathcal{C}(f ; x)=\bigcap\{\mathrm{Cl}(f(U)): U \in \Upsilon(x)\}
$$

where $\mathrm{Cl}(U)$ denotes the closure of $U$. Weston [6] observed that if $Y$ is a Hausdorff space and $f$ is continuous, then $\mathcal{C}(f ; x)$ is degenerate. He also noted that the converse holds provided $Y$ is compact, and, in general, does not hold if $Y$ is not compact. In this paper we take $Y$ to be either $H$-closed, minimal Hausdorff, minimal Urysohn, or minimal regular, and use $\mathcal{C}(f ; x)$ as a tool to obtain information about the continuity of $f$.

2. Preliminaries. In this section we give some basic definitions and establish two lemmas that are useful in the sections which follow.

Let $X$ be a topological space. An open subset $U$ of $X$ is said to be regular open [3, p. 92] if $U=\operatorname{Int}(\mathrm{Cl}(U))$, where Int denotes the interior operator. A subset $F$ of $X$ is said to be regular closed [3, p. 92] if $F=$ $\mathrm{Cl}(\operatorname{Int}(F))$. If $f$ is a function from $X$ into a topological space $Y, f$ is said to be closed (almost closed) if $f(K)$ is closed in $Y$ for every closed (regular closed) set $K$ in $X$. We say $f$ is an open mapping if $f(V)$ is open in $Y$ for every open set $V$ in $X$.

Lemma 1. Let $f$ be a closed map from a regular space $X$ into a space $Y$. If $f^{-1}(y)$ is closed in $X$ for every $y \in Y$, then $\mathcal{C}(f ; x)$ is degenerate for every $x \in X$.

Received by the editors June 28, 1974 and, in revised form, October 4, 1974. AMS (MOS) subject classifications (1970). Primary 54D25; Secondary 54C10. Key words and phrases. Almost continuous, cluster set, $H$-closed, minimal Hausdorff, minimal regular, open filterbase, regular closed. 
Proof. The proof follows easily from the observation $\mathcal{C}(f ; x)=$ $\bigcap\{f(\mathrm{Cl}(U)): U \in \Re(X)\}$.

Lemma 2. Let $f$ be an almost closed injection from a Hausdorff space $X$ into a space $Y$, then $\mathcal{C}(f ; x)$ is degenerate for every $x \in X$.

Proof. The proof follows easily from the well-known fact [3, p. 92] that the closure of an open set is regular closed and the observation $\mathcal{C}(f ; x)$ $\subseteq \bigcap\{f(\mathrm{Cl}(U)): U \in \eta(x)\}$.

3. H-closed and minimal Hausdorf spaces. Let $f$ be a function from a space $X$ into a space $Y$. We say $f$ is almost continuous [4, Definition 3] at $x \in X$ if for every regular open nbd $V$ of $f(x)$, there exists a $W \in \Re(x)$ such that $f(W) \subseteq V$.

Theorem 3.1. Let $f$ be an open mapping from a space $X$ into an $H$ closed space $Y$, and let $x \in X$. Then $\mathcal{C}(f ; x)$ is degenerate if and only if $f$ is almost continuous at $x$.

Proof. Necessity. Let $V$ be a regular open nbd of $f(x)$. Suppose $f(U) \cap(Y-V)$ is nonempty for every nbd $U$ of $x$. Note that $(Y-V)$ is a regular closed subset of $Y$, and hence is $H$-closed [7, p. 127]. Now $\{\mathrm{Cl}(f(U)) \cap(Y-V): U \in \Upsilon(x)\}$ is a family of closed sets, and their interiors with respect to $(Y-V)$ satisfy the finite intersection property. Thus $\mathcal{C}(f ; x) \cap(Y-V)$ is nonempty and therefore $\mathcal{C}(f ; x)$ is not degenerate.

Sufficiency. Assume $f$ is almost continuous at $x$ and let $\tau$ denote the topology on $Y$. Let $\tau_{s}$ denote the topology on $Y$ generated by the regular open sets of $\tau$. Now we have

$$
\{f(x)\}=\bigcap\left\{\mathrm{Cl}_{\tau_{s}}(f(U)): U \in \Re(x)\right\} \supseteq \bigcap\left\{\mathrm{Cl}_{\tau}(f(U)): U \in \Re(x)\right\} \supseteq \mathcal{C}(f ; x) .
$$

Hence $\mathcal{C}(f ; x)=\{f(x)\}$ is degenerate.

Before stating the next theorem, we should point out that an example of an $H$-closed Urysohn space which is not compact may be found in [1, Example 3.13]. A function $f$ from a space $X$ into a space $Y$ is said to be connected [5] if $f(C)$ is connected in $Y$ for every connected subset $C$ of $X$.

Theorem 3.2. If $f$ is an open connected mapping from a locally connected space $X$ into an H-closed Urysohn space $(Y, \tau)$, then $f$ is almost continuous at $x \in X$ if and only if $\varrho(f ; x)$ is countable.

Proof. Sufficiency. Assume $\mathcal{C}(f ; x)$ is countable. Let $\mathcal{C}(x)$ be a nbd base of open connected sets at $x$. Note that $\mathrm{Cl}_{\tau}(f(U))$ is a regular closed connected set for every $U \in \mathcal{C}(x)$. Let $\tau_{s}$ denote the topology on $Y$ generated by the regular open sets of $\tau$. 3y Theorem 3.4(b) of [1], $\left(Y, \tau_{s}\right)$ is compact. Thus $\left\{\mathrm{Cl}_{\tau}(f(U)): U \in \bigodot(x)\right\}$ is a collection of $\tau_{s}$ continua directed 
by inclusion, which implies $\mathcal{C}(f ; x)$ is a $\tau_{s}$ continuum [7, Theorem 28.2, p. 203]. Consequently, $\mathcal{C}(f ; x)$ must be either a single point or uncountable. Our assumption that $\mathcal{C}(f ; x)$ is countable implies $\mathcal{C}(f ; x)$ is a single point and, therefore, $f$ is almost continuous by Theorem 3.1.

Necessity. Theorem 3.1.

We now focus our attention on minimal Hausdorif spaces, and at the end of this section state a theorem which gives results for $H$-closed and minimal Hausdorff spaces in combined form.

Theorem 3.3. Let $f$ be an open mapping from a space $X$ into a minimal Hausdorff space $Y$. Then $f$ is continuous at $x \in X$ if and only if $\mathcal{C}(f ; X)$ is degenerate.

Proof. We have only to show sufficiency. Assume $\mathcal{C}(f ; x)$ is degenerate, and let $f(\eta)=\{f(U): U \in \eta(x)$ and $U$ is open $\}$. Now $f(\gamma)$ is an open filterbase, and the assumption $\mathcal{C}(f ; x)$ is degenerate implies $f(\gamma)$ has a unique adherent point which must be $f(x)$. Since $Y$ is minimal Hausdorff, $f(\eta)$ converges to $f(x)$ and therefore $f$ is continuous.

We are now ready to apply Lemmas 1 and 2 .

Theorem 3.4. Let $f$ be an open mapping from a space $X$ into a minimal Hausdorff (H-closed) space $Y$.

(1) If $X$ is regular, $f$ is closed, and $f^{-1}(y)$ is closed for every $y \in Y$, then $f$ is continuous (almost continuous).

(2) If $f$ is an almost closed injection and $X$ is Hausdorff, then $f$ is continuous (almost continuous).

Proof. (1) Lemma 1 and Theorem 3.3 (Theorem 3.1).

(2) Lemma 2 and Theorem 3.3 (Theorem 3.1).

Corollary 3.5. Every continuous bijection from an H-closed space onto a Hausdorff space has an almost continuous inverse.

Proof. The map $f^{-1}$ is an open closed injection from a Hausdorff space into an $H$-closed space. Now apply Theorem 3.4(2).

4. Minimal Urysohn and minimal regular spaces. In order to be consistent with [1], our definition of regular (in this section only) includes the $T_{1}$ separation property. An open filterbase $B$ in a space is called a Urysohn filterbase if for each point $p \notin \bigcap\{\mathrm{Cl}(B): B \in B\}$, there is an open nbd $U$ of $p$ and $B \in B$ such that $\mathrm{Cl}(U) \cap \mathrm{Cl}(B)=\varnothing$. An open filterbase $U$ is called a regular filterbase if for each $U \in U$ there exists a $V \in U$ such that $\mathrm{Cl}(V)$ $\subseteq U$. Note that a regular filterbase is a Urysohn filterbase. The following theorem gives a filterbase characterization of minimal regular and minimal Urysohn spaces. 
Theorem 1.1 [1]. A regular (Urysohn) space is minimal regular (Urysohn) if and only if every regular (Urysohn) filterbase with a unique adherent point converges.

Theorem 4.2. Let $f$ be an open and closed mapping from a regular space $X$ into a minimal regular (Urysohn) space $Y$. Then / is continuous at $x \in X$ if and only if $\mathcal{P}(f ; x)$ is degenerate.

Proof. We need only show sufficiency. Observe that for $x \in X,\{f(U)$ : $U \in Y(x)\}$ is a regular filterbase (and hence a Urysohn filterbase) and apply Theorem 4.1.

We are now ready for another application of Lemma 1.

Theorem 1.3. Let $f$ be an open and closed mapping from a regular space $X$ into a minimal regular (Urysohn) space $Y$. Then $f$ is continuous if and only if the preimages of points in $Y$ are closed in $X$.

Proof. Necessity is obvious and sufficiency follows from Lemma 1 and Theorem 4.2.

Acknowledgement. The author is indebted to his major professor, Paul E. Long, for his patience, guidance, and constructive criticism.

\section{REFERENCES}

1. M. P. Berri, J. R. Porter and R. M. Stephenson, Jr., A survey of minimal topological spaces, General Topology and its Relations to Modern Analysis and Algebra, III (Proc. Conf., Kanpur, 1968), Academia, Prague, 1971, pp. 93-114. MR 43 \#3985.

2. N. Bourbaki, Elements of mathematics. General topology. Part 1, Hermann, Paris; Addison-Wesley, Reading, Mass., 1966, MR 34 \#5044a.

3. J. Dugundji, Topology, Allyn and Bacon, Boston, Mass., 1966. MiR 33 \#1824.

4. Paul E. Long and Donald A. Carnahan, Comparing almost continuous func= tions, Proc. Amer. Math. Soc. 38 (1973), 413-418. MR 46 \#9922.

5. William J. Pervin and Norman E. Levine, Connected mappings of Hausdorff spaces, Proc. Amer. Math. Soc. 9 (1958), 488-496. MR 20 \#1970.

6. J. D. Weston, Some theorems on cluster sets, J. London Math. Soc. 33 (1958), 435-4.11. MR $20 \# 7109$.

7. Stephen Willard, General topology, Addison-W'esley, Reading, Mass., 1970. MR $41 \# 9173$.

DEPARTMENT OF MATHEMATICS, UNIVERSITY OF ARK ANSAS, FAYETTEVILLE, ARK ANSAS 72701 